\title{
Human Body Effect on Static UWB WBAN Off-Body Radio Channels
}

\author{
Timo Kumpuniemi ${ }^{[0000-0003-4047-764 X]}$, Juha-Pekka Mäkelä, Matti Hämäläinen, \\ Kamya Yekeh Yazdandoost and Jari Iinatti \\ Centre for Wireless Communications, University of Oulu, Finland \\ timo.kumpuniemi@oulu.fi
}

\begin{abstract}
This paper presents the effect of a human body on ultra wideband offbody wireless body area network radio channels. The work is based on static measurements in an anechoic chamber by using a vector network analyzer in a 2-8 GHz frequency band. Thirteen antenna locations on a human body are used while the off-body node is attached to a pole at a distance one and two meters from the test subject. Two planar prototype antennas are applied: dipole and double loop. The data analysis is carried out in time domain by observing the first arriving signal components of the channel impulse responses. The classical path loss model fitting results path loss exponents of 1.7 and 1.4 for the dipole and double loop, respectively. The classical path loss model is not found to be suitable in all cases in wireless body area networks as the path loss exponent varies greatly depending on the antenna site under examination. The absolute path losses reach values between 50.6...66.5 dB (dipole) and 49.9 ..68.2 dB (double loop) depending on the antenna location. In most of the cases the double loop performs better than the dipole. When averaged over all antenna sites the mean path losses lie in the range of $57.6 \ldots 61.3 \mathrm{~dB}$ and their standard deviation approx. $4 . .5 \mathrm{~dB}$ depending on the distance and antenna focus.
\end{abstract}

Keywords: Shadowing, Ultra Wideband, Wireless Body Area Network.

\section{Introduction}

The development in computing power of electronic devices together with their decreasing sizes and energy efficiency have enabled the birth of new application areas to wireless appliances. Currently one key focal areas in wireless communications is the fifth generation $(5 \mathrm{G})$ systems and its implementation into reality. One vertical in $5 \mathrm{G}$ systems is the concept of internet of things (IoT) where the number of different kind of sensors utilized in new environments is expected to explode. If the sensors are operating wirelessly, one or several of them are usually planned to be in contact to an access point. IoT sensors can be installed into, e.g., vehicles, buildings, house appliances, industrial plants and machines. Furthermore, IoT sensors can be utilized with humans thus forming a wireless body area network (WBAN). With persons, WBANs can be constructed by applying sensors on human bodies (on-body), in the close vicinity of them (off- 
body) or even inside the bodies (in-body). In body-to-body communications the sensors are exchanging information between two or more individuals [1].

WBANs can be applied in various applications. They can be used in sports to monitor the body parameters providing information on the effectiveness of the exercise. In the well-being sector, the daily activity can be monitored as well as, e.g., the quality of the sleep. Authorities, as fire and police departments or the military, can utilize WBANs in their daily work to increase their own safety.

One of the most enhancing and promising areas is however the medical field. A practical reason to this is the constant ageing of population in many nations around the world forcing to search for new more effective and economical solutions to provide healthcare services to the growing number of patients. Different technological solutions are one answer to this question, among them belonging also WBANs. The wireless characteristics of WBANs enable better working comfortability and effectiveness for the medical staff. The patients will have a more pleasant treatment experience as they can move freely. They may even live their lives during the recovery phase at homes through remote monitoring the vital parameters from the hospital or other medical unit.

The ultra wideband (UWB) technology is a very suitable solution for wireless communications to use in the WBANs for many reasons. The wide bandwidth of the UWB signals facilitate an accurate positioning and provide a good performance in difficult radio channel conditions with a large number of multipath components. The transmission power of UWB signals is low minimizing the interference caused to other systems. In practice, the UWB transmission power spectral densities often remain below the existing noise power density. On the other hand, UWB is robust against interferences from other transmissions originating nearby. The transceiver structures are simple providing a low unit price, a small size and a low power consumption providing excellent battery life $[2,3]$.

The adoption of UWB technology in WBANs has been under investigation for over a decade [4]. Most of published articles in the field of radio channels are related to onbody communications [5-7] both in static and dynamic cases. Off-body WBANs are often covered in narrowband cases [8,9], but for UWB signals references are found as well [10-13].

This paper reports static UWB off-body radio channel measurements with the focus on the effect of the human body on the channels. The measurements are conducted in an anechoic chamber to exclude the radio channel responses originating beyond the human body. A vector network analyzer (VNA) is applied in the frequency sweeping mode to examine the $2-8 \mathrm{GHz}$ frequency band. The results extend the knowledge on the off-body UWB channels beyond the ones reported in [13] to the direction of static UWB channels. The work is a part of a larger measurement campaign reported, e.g., in $[6,7,14,15]$.

The structure of the paper is the following. In Section 2, the measurement setup is explained. Section 3 describes the measurement scenario with antenna locations and practical arrangements of the measurements. Section 4 explains the data processing methods together with the presentation of the results. Conclusions and future work plans are covered in Section 5. 


\section{Measurement Setup}

\subsection{Test Person and Anechoic Chamber}

A $183 \mathrm{~cm}$ tall male test person with a $95 \mathrm{~kg}$ body weight was standing still in an anechoic chamber facing towards the off-body antenna pole. The chamber had a floor size of $245 \mathrm{~cm}$ by $365 \mathrm{~cm}$ and it was assembled by using movable absorber blocks. The test person was wearing a cotton T-shirt and jeans. All metal containing items and shoes were absent during the measurements.

\subsection{Equipment}

A four-port VNA (Rohde\&Schwarz ZVA-8, upper frequency limit $8 \mathrm{GHz}$ ) was located outside the anechoic chamber and four eight meters long measurement cables were led into the chamber. The VNA was sweeping the 2-8 GHz frequency band 100 times with 1601 points within the band in each measurement. A more detailed list of the VNA settings can be found in [14].

Two planar prototype antenna types were utilized in the measurements: dipole and double loop. The maximum total free space gains of the antennas is $6 \mathrm{dBi}$. The operational frequency band for the antennas is $2-12 \mathrm{GHz}$. The detailed description of the structures and the performance of the antennas, as well as the measured free space radiation patterns are found in [16-18].

\section{Measurement Scenario}

Thirteen antenna locations were selected in different parts of the human body: the locations represent either existing or very probable sites for the on-body sensors. The offbody antenna was located on a pole at the height of two meters. The measurements were repeated with the human-pole distances of one and two meters and with both antenna types, respectively.

As depicted in Fig. 1, three antenna spots (1-3) are situated at the head, six at the limbs $(6,7,9,11-13)$ and four on the torso $(4,5,8,10)$. The antennas were attached three at a time on the body- due to the four-port VNA- by using elastic bands and paper tape while the fourth one remained at the pole. A $20 \mathrm{~mm}$ piece of ROHACELL HF31 material was inserted to keep the antenna-body distance constant between the antennas and the body. In [17], this distance was noted to be a proper choice providing good antenna matching and channel gain characteristics close to the free space performance of the antennas. 


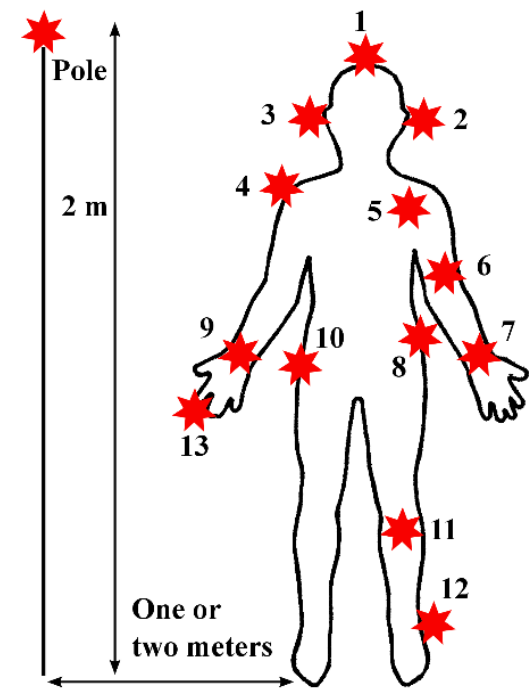

Fig. 1. The antenna locations used in the measurements. The case is measured with two humanpole distances: one and two meters.

\section{$4 \quad$ Results}

\subsection{Data Processing}

The results are extracted from the channel impulse responses (CIRs) in the time domain. By this manner, the possible reflection components due to the un-idealities of the anechoic chambers can be filtered out. The CIRs are obtained from the frequency domain measurement data by applying the inverse fast Fourier transform. No windowing is applied in the transform. From the CIRs, the first arriving paths (FAPs) are considered in the calculations, i.e., considering the first multipath component. In the measurement scenario it was noted that other multipaths are weak compared to FAP originating mainly from reflections from the body. As an anechoic chamber is used, the reflections from other sources than the body were also noted to be minor. However, utilizing more multipaths in a RAKE-type receiver would be beneficial in real life situations, where FAP may not be necessarily the strongest CIR component. The FAPs are found out by detecting all arriving multipath components above a threshold and selecting the first component. The threshold is set to be $10 \mathrm{~dB}$ lower than the strongest signal component, since this value was noted to be a suitable choice to block the noise and rising edge spikes of the CIRs.

The full-band of the measurements was selected for the data processing, as the division for high- and low bands in the standard IEEE 802.15.6 [19] was not feasible due to the frequency band limitation of the applied VNA. 


\subsection{Path Loss Models Between the Antenna Sites}

At first, the path losses (PLs) are compared between the different antenna sites. The PL can be expressed as

$$
P L(d)=P L\left(d_{0}\right)+10 n \log _{10}\left(d / d_{0}\right)+S,
$$

where $P L(d)$ is the path loss in decibels at the distance of $d, P L\left(d_{0}\right)$ is the path loss at the used reference distance $d_{0}(50 \mathrm{~mm}), n$ is the path loss exponent and $S$ is a random scattering term. In the measurements in the paper, the effect of $S$ is minor as the case is a static one measured in an anechoic chamber. The path distances $d$ are solved from the CIRs based on the arrival time of the FAPs. The signal propagation speed in the antennas and the physical dimensions of the antennas cause delay bringing out inaccurate distance values. The explanation is as follows. After calibrating the VNA it is assumed that the zero delay plane is at the open ends of the measurement cables, i.e., if the cables are connected together, they show a zero delay in the time domain. When antennas are applied, two phenomenon occur. Firstly, the distance between the cable ends and the phase center of the antennas cause delay, both in transmit and receive side. Secondly, the electrical parameters of the antenna parameters have an effect on the signal propagation speed $v$. In a lossless case, it can be stated with the well-known classical equation as:

$$
v=c / \sqrt{\varepsilon_{\mathrm{r}}},
$$

where $c$ is the propagation of light in vacuum, and $\varepsilon_{\mathrm{r}}$ is the relative permittivity of the media, i.e., in this case the substrate material of the antenna structures. Also this effect takes place both in transmit and receive antennas.

Therefore, a correction factor is needed for this reason: the correction factors of 90 $\mathrm{mm}$ and $109 \mathrm{~mm}$ in distance are applied for the dipole and double loop cases, respectively. They were obtained by inserting the antennas to a carefully measured fixed distance, and the measured the distance error in six relative positions to each other, for both antenna types. The correction factors are the mean values of the noted error distances in the measured positions. The effect is described in more detail in [14].

Table 1 presents the numerical values of $n$ and $P L\left(d_{0}\right)$ based on the first order least squares data fitting. The results are shown for the antenna sites separately and for both antenna types. The case "All" depicts the general case with all antenna locations considered jointly in the analysis. The path loss exponent is on the average 1.7 for the dipole antenna varying between the values $-0.7 \ldots 4.7$. For the double loops, the path loss exponent reaches the value 1.4 on the average. The range of variation is $-4.2 \ldots 1.6$. The negative values originate from firstly the shadowing effect caused by the human body. In different measurements, the test person may be situated slightly differently with respect to the off-body antenna on the pole. Therefore, the path loss is more dependent on the degree of shadowing instead of the distance. Secondly, the antennas have been reported to have up to $30 \mathrm{~dB}$ variation in gain depending on the direction of radiation and the frequency [18] having a strong impact on the received signal power 
with only a minor change of antenna position. The effect of body shadowing and antenna radiation pattern on the classical path loss model (1) is noted earlier in [6]. The suitability of classical path loss model in WBAN cases is questioned also in [20].

Path loss exponent values between 1.28...3.90 have been reported in [12] for an offbody UWB case in an echoic surroundings. Therefore, the average results in Table 1 are in line with the ones in [12]. The path loss exponent values below two are due to the human body acting as a directing element for the electromagnetic radiation.

Table 1. Path loss model values of the antenna sites.

\begin{tabular}{lllll}
\hline & Dipole & & \multicolumn{2}{l}{ Double loop } \\
\hline Site & $n$ & $P L\left(d_{0}\right)[\mathrm{dB}]$ & $n$ & $P L\left(d_{0}\right)[\mathrm{dB}]$ \\
1 & 1.65 & 32.8 & 0.9 & 50.3 \\
2 & 2.5 & 16.7 & 1.6 & 29.6 \\
3 & 1.4 & 40.4 & 1.4 & 34.5 \\
4 & 2.0 & 25.9 & 1.5 & 30.4 \\
5 & 2.3 & 27.8 & 1.4 & 30.4 \\
6 & 1.6 & 28.8 & 0.8 & 38.9 \\
7 & 4.7 & -16.3 & 0.9 & 39.1 \\
8 & -0.7 & 66.9 & -0.7 & 67.1 \\
9 & 1.5 & 40.8 & -0.9 & 68.9 \\
10 & 1.7 & 29.5 & 0.5 & 45.6 \\
11 & 2.9 & 17.5 & -2.4 & 101.4 \\
12 & -0.4 & 66.8 & -4.2 & 138.0 \\
13 & 2.8 & 10.6 & -2.4 & 97.0 \\
All & 1.7 & 32.4 & 1.4 & 35.3 \\
\hline
\end{tabular}

\subsection{Absolute Path Loss}

Next, the absolute path loss values are examined. Table 2 gathers together the obtained results for all antenna locations, both antenna types and for both measured distances. The values are extracted by averaging measured sweeps for each measurement separately. The PLs range between 50.6..66.5 dB and 49.9..68.2 $\mathrm{dB}$ for the dipole and double loop antenna cases respectively. When comparing the 13 antenna location cases and both measured distances between the antennas, in 17 cases out of 26 the double loop antenna results lower PL than the dipole. At the locations 3, 4, 5, 6, 7, 9 and 10 the double loop performs better at both distances. At the sites 1, 8 and 12 the dipole antenna shows lower PL values. Based on the comparison it can be stated that the double loop antenna outperforms statistically in most cases the dipole.

Table 3 reports the mean $(\mu)$ values of the PLs and their standard deviation $(\sigma)$ in decibels. The results are shown for both antennas and distances. Also the cases where both distances are joined and both antenna data are joined are presented. The mean 
value is calculated from the data in Table 2 by averaging the PLs in linear scale and converting the result in $\mathrm{dB}$. The standard deviations are obtained from the decibel valued PLs.

The mean values lie between $57.6 \ldots 61.3 \mathrm{~dB}$. The standard deviations vary approx. between $4 \ldots 5 \mathrm{~dB}$. When both distances are examined, the double loop performs slightly better confirming the observation noted from Table 2. The PL for the double loop is lower at two meters compared with the one meter case, which is due to the dominating body shadowing and antenna radiation patter effect over the distance dependence as discussed in Subsection 4.2.

Table 2. Absolute measured path losses [dB].

\begin{tabular}{lllll}
\hline & Dipole & & Double loop & \\
\hline Site & One meter & Two meters & One meter & Two meters \\
1 & 54.9 & 59.5 & 61.3 & 64.5 \\
2 & 50.6 & 57.2 & 51.8 & 56.1 \\
3 & 59.7 & 63.4 & 53.9 & 57.6 \\
4 & 54.7 & 59.3 & 51.4 & 54.9 \\
5 & 60.0 & 65.4 & 49.9 & 53.3 \\
6 & 52.2 & 55.7 & 49.9 & 51.7 \\
7 & 54.7 & 62.0 & 53.1 & 54.8 \\
8 & 57.0 & 55.7 & 57.1 & 55.9 \\
9 & 63.1 & 66.5 & 56.3 & 54.7 \\
10 & 54.8 & 57.7 & 53.4 & 54.4 \\
11 & 62.5 & 66.2 & 64.3 & 61.1 \\
12 & 60.5 & 60.0 & 68.2 & 63.5 \\
13 & 53.4 & 57.7 & 60.6 & 56.8 \\
\hline
\end{tabular}

Table 3. Mean and standard deviations of the path losses [dB].

\begin{tabular}{llll}
\hline & & $\mu$ & $\sigma$ \\
\hline \multirow{3}{*}{ Dipole } & One meter & 57.6 & 4.0 \\
& Two meters & 61.3 & 3.9 \\
& Both distances & 59.6 & 4.3 \\
\hline \multirow{2}{*}{ Double } & One meter & 58.2 & 4.8 \\
loop & Two meters & 57.8 & 3.9 \\
& Both distances & 58.0 & 4.8 \\
\hline \multirow{2}{*}{ Both } & One meter & 57.9 & 4.9 \\
antennas & Two meters & 59.7 & 4.2 \\
& Both distances & 58.9 & 4.6 \\
\hline
\end{tabular}




\section{$5 \quad$ Conclusion and Future Work}

The human body effect on the UWB WBAN off-body radio channels are discussed. The work is based on static measurements with a VNA set to the frequency sweeping mode at 2-8 GHz. To limit the examination on the effects originating from the human body, the measurements are conducted in an anechoic chamber. Thirteen antenna locations are selected on the body while the off-body antenna is attached to a pole at one or two meter distances. The experiment is repeated with prototype dipole and double loop antennas.

By applying the classical path loss model the average path loss exponent is found to be 1.7 (dipole) and 1.4 (double loop). The obtained path loss exponents are noted to variate largely depending on the antenna site under study. The negative exponents in some cases are a result of the dominance of the body shadowing together with the antenna pattern variation over the distance. The classical path loss model is thus found to be not always unconditionally suitable in the WBAN cases where the channel model may be rather shadowing and radiation pattern dependent than distance dependent.

The absolute path losses reach numerical values between 50.6...66.5 $\mathrm{dB}$ (dipole) and $49.9 \ldots 68.2 \mathrm{~dB}$ (double loop). In the majority of cases, when examining individually the antenna spots and distances, the double loop performs slightly better than the dipole. When examining the antenna sites jointly, and/or the different distances and antenna types together, the mean values of the path losses are found to be between $57.6 \ldots 61.3 \mathrm{~dB}$ and the standard deviation approx. $4 \ldots 5 \mathrm{~dB}$.

As a future work, it would be interesting to extend the measurements into echoic environments as well as to increase the number of measured distances and antenna sites in order to obtain more data for the data analysis. The frequency range could be extended to cover the full frequency range defined by the IEEE 802.15.6 standard for UWB by adopting a VNA with higher upper frequency limit. Furthermore, a larger set of test persons could be applied in order to investigate, e.g., the effect of the test person age, gender and the different body parameters.

\section{Acknowledgement}

This research has been financially supported in part by Academy of Finland 6Genesis Flagship (grant 318927).

\section{References}

1. Hall, P.S., Hao, Y.: Antennas and propagation for body-centric wireless communications. 2nd edn. Artech House, Norwood, MA, USA (2012).

2. Oppermann, I., Hämäläinen, M., Iinatti, J. (eds.): UWB theory and applications. John Wiley \& Sons, West Sussex, England (2004).

3. Ghawami, M., Michael, L.B., Kohno, R.: Ultra wideband signals and systems in communication engineering, 2nd edn. John Wiley \& Sons, West Sussex, England (2007). 
4. Fort, A., Desset, C., De Doncker, P., Wambacq, P., Van Biesen, L:, An ultra-wideband body area propagation channel model-from statistics to implementation, IEEE Transactions on Microwave Theory and Techiques, 54(4), 1820-1826 (2006).

5. Di Bari, R., Abbasi, Q. H., Alomainy, A., Hao, Y.: An advanced UWB channel model for body-centric wireless networks. Progress In Electromagnetics Research, 136, 79-99 (2013).

6. Kumpuniemi, T., Hämäläinen, M., Yekeh Yazdandoost, K., Iinatti, J.: Categorized UWB onbody radio channel modeling for WBANs. Progress in Electromagnetic Research B, 67, 1-16 (2016).

7. Kumpuniemi, T., Hämäläinen, M., Yekeh Yazdandoost, K, Iinatti, J.: Human body shadowing effect on dynamic UWB on-body radio channels. IEEE Antennas and Wireless Propagation Letters, 16, 1871-1874 (2017).

8. Rosini, R., D'Errico, R.: Off-body channel modelling at $2.45 \mathrm{GHz}$ for two different antennas. In: Proceedings of the 6th European Conference on Antennas and Propagation (EUCAP). 3378-3382, IEEE, Prague, Czech Republic (2011).

9. Yoo, S. K., Cotton, S. L.: Shadowed fading in indoor off-body communications channels: a statistical characterization using the $\kappa-\mu$ /gamma composite fading model. IEEE Transactions on Wireless Communications, 17(8), 5231-5244 (2016).

10. Ali, A. J., Scanlon, W. G., Cotton, S. L.: Pedestrian effects in indoor UWB off-body communication channels. In: Proceedings of. 2010 Loughborough Antennas \& Propagation Conference, 57-60, IEEE, Loughborough, UK (2010).

11. Catherwood, P. A., Scanlon, W. G.: Body-centric antenna positioning effects for off-body UWB communications in a contemporary learning environment. In: Proceedings of the 8th European Conference on Antennas and Propagation (EUCAP), 1571-1574, IEEE, The Hague, Netherlands (2014).

12. Garcia-Serna, R.-G., Garcia-Pardo, C., Molina-Garcia-Pardo, J.: Effect of the receiver attachment position on ultrawideband off-body channels. IEEE Antennas and Wireless Propagation Letters, 14, 1101-1104 (2015).

13. Kumpuniemi, T., Mäkelä, J-P., Hämäläinen, M., Yekeh Yazdandoost, K., Iinatti J.: Dynamic UWB off-body radio channels - human body shadowing effect. In: Proceedings of the 28th Annual IEEE International Symposium on Personal, Indoor and Mobile Radio Communications (IEEE PIMRC 2017), 1-7, IEEE, Montreal, Canada (2017).

14. Kumpuniemi, T., Tuovinen, T., Hämäläinen, M., Yekeh Yazdandoost, K., Vuohtoniemi, R., Iinatti, J.: Measurement-based on-body path loss modelling for UWB WBAN communications. In: Proceedings of the $7^{\text {th }}$ International Symposium on Medical Information and Communication Technology (ISMICT), 233-237, IEEE, Tokyo, Japan (2013).

15. Kumpuniemi, T., Hämäläinen, M., Tuovinen, T., Yekeh Yazdandoost, K. Iinatti, J., Generic small scale channel model for on-body UWB WBAN communications. In: Proceedings of The Second Ultra Wideband for Body Area Networking Workshop (UWBAN-2013) Co-located with the 8th International Conference on Body Area Networks (BodyNets-2013), 370-374, ACM, Boston USA (2013).

16. Tuovinen, T., Kumpuniemi, T., Yekeh Yazdandoost, K., Hämäläinen, M., Iinatti, J.: Effect of the antenna-human body distance on the antenna matching in UWB WBAN applications. In: Proceedings of the $7^{\text {th }}$ International Symposium on Medical Information and Communication Technology (ISMICT), 193-197, IEEE, Tokyo, Japan (2013).

17. Tuovinen, T., Kumpuniemi, T., Hämäläinen, M., Yekeh Yazdandoost, K., Iinatti, J.: Effect of the antenna-body distance on the on-ext and on-on channel link path gain in UWB WBAN applications. In: Proceedings of the 35th Annual International Conference of IEEE Engineering in Medicine and Biology Society (EMBC), 1242-1245, IEEE, Osaka, Japan (2013). 
18. Kumpuniemi, T., Hämäläinen, M., Yekeh Yazdandoost, K., Iinatti, J.: Measurements for body-to-body UWB WBAN radio channels. In: Proceedings of the 9th European Conference on Antennas and Propagation (EUCAP), 1-5, IEEE, Lisbon, Portugal (2015).

19. IEEE Standard for Local and Metropolitan Area Networks, IEEE 802.15.6-2012 - Part 15.6: Wireless Body Area networks, 2012

20. Smith, D. B., Miniutti, D., Lamahewa, T. A., Hanlen, L. W.: Propagation models for bodyarea networks: a survey and new outlook. IEEE Antennas and Propagation Magazine, 55(5), 97-117 (2013). 